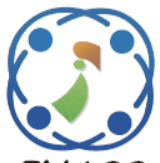

\title{
CLIF: Cross-Layer Interactive Framework for Enhancing Network Lifetime and Communication in WSN
}

\author{
Shoba Chandra $^{1^{*}} \quad$ Kiran Kumari Patil $^{2} \quad$ Suresha Talanki $^{3}$ \\ ${ }^{1,2}$ REVA University, Bangalore, Karnataka, India \\ ${ }^{1,3}$ Sri Venkateshwara College of Engineering, Bangalore, Karnataka, India \\ * Corresponding author's Email: shoba.m.ise@svcengg.com
}

\begin{abstract}
Adoption of Cross-Layer based techniques in Wireless Sensor Network (WSN) has been reported to be an efficient solution towards improving energy efficiency, scheduling related problems among the resource-limited sensor nodes. The existing approaches are reviewed to ensure that there exists further scope for the improvement and enhancement in terms of resolving the issues associated with even faster interaction among the layers of the conventional protocol stack for catering up and facilitating the demands of faster response time in real-time applications. Therefore, Cross Layer Interactive Framework (CLIF) a novel and advanced approach is proposed for essentially sustaining symmetry between the crucial performance parameters like data delivery and energy efficiency for a given dynamic communication circumstances in WSN. In addition, to this message prioritization is incorporated in CLIF for processing those messages with high degree of significance. Predominantly CLIF can be used for the diverse time critical and mission critical IoT applications. The results demonstrate that the proposed CLIF scheme offers better energy efficiency and enhanced data delivery services in WSN when compared with the existing cross-layer approaches.
\end{abstract}

Keywords: Cross-layer, Energy efficiency, Protocol stack, MAC layer, Network layer, CLIF.

\section{Introduction}

The problems associated with Wireless Sensor Network (WSN) have been investigated since more than a decade addressing multiple problems. The common problems associated with a sensor node are i) limited memory, ii) limited power supply, iii) limited computational capability, etc [1]. The protocol stack in WSN is essentially design to frame up its characteristics behaviour and hence the design of protocol stack is highly questionable in order to obtain better communication performance. There are various researchers since 2002 to offer multiple definition of protocol stack in WSN, but in reality, there have been various studies already conducted that can switch to any new form and order among the layers differently from the conventional protocol stack in WSN [2]. It mainly consists of physical layer at the bottom followed by data link layer, network layer, transport layer, and application layer at the top [3]. The conventional stack formulation of WSN also discusses about the existence of various form of management planes like Quality of Service (QoS) management, Task management, Security management, Mobility management, and Power management [4].

The base layer i.e. Physical layer is responsible for offering the information related to modulation, distance of hop, power of forwarding, etc. The second layer after physical layer is data link layer that performs multiplexing of various streams of environmental aggregated sensory data, Medium Access Control (MAC), and detection of data frame. Majority of the routing using data-centric approach and energy conservation is carried out in MAC layer itself. However, from numerous studies in WSN, it has been found that existing MAC layers in WSN is not much capable of maximum power retention. The third layer is Network layer which is mainly responsible for executing routing protocols and 
making decision about routing operation. The fourth layer is Transport layer which is responsible for carrying out all sorts of traffic management operation e.g. Reliability, Congestion Control, resisting dropping of packets, etc. The final layer is Application layer that emphasizes implementation of specific norms of application demands. The conventional layer wise interaction can be modified by implying cross-layer approach where the order and form of internal communication among the layers in protocol stack of WSN can be modified as per requirement.

The conventional layered protocol stack for WSN is illustrated in Fig. 1. The biggest challenge in this direction of research work is to ensure energy efficiency as there is a considerable depletion of energy between the base station and the sensor node when they opt for cross-layer based design. Not only this, it may also offer a significant challenge in controlling the channel capacity usage that finally results in formation of control overheads. At present, there has been various studies [5] where various cross layer approaches have been implemented for addressing multiple problems in WSN; however, there are some significant loopholes in almost majority of the research approaches i) lesser benchmarking work leads to uncertain rate of successful applicability in real-time application, ii) less balance between energy conservation and data delivery performance, iii) inclusion of significant overheads leads to computational complexity, iv) less focus on message replications during both broadcasting and scheduling, and v) lack of message prioritization.

Therefore, we present a novel cross layer interactive framework that is exclusively meant for addressing such unsolved problems in WSN in order to cater up the demands of real-time applications under dynamic environment. An analytical modelling-based approach is presented in order to develop the proposed cross-layer interactive framework in WSN. Section 1.1 discusses about the existing literatures where different cross-layer based techniques are discussed for solving multiple energy-related problems followed by discussion of research problems in Section 1.2 and proposed solution in 1.3. Section 2 discusses about algorithm implementation emphasizing on message ranking and energy efficiency in WSN followed by discussion of result analysis in Section 3. Finally, the conclusive remarks are provided in Section 4.

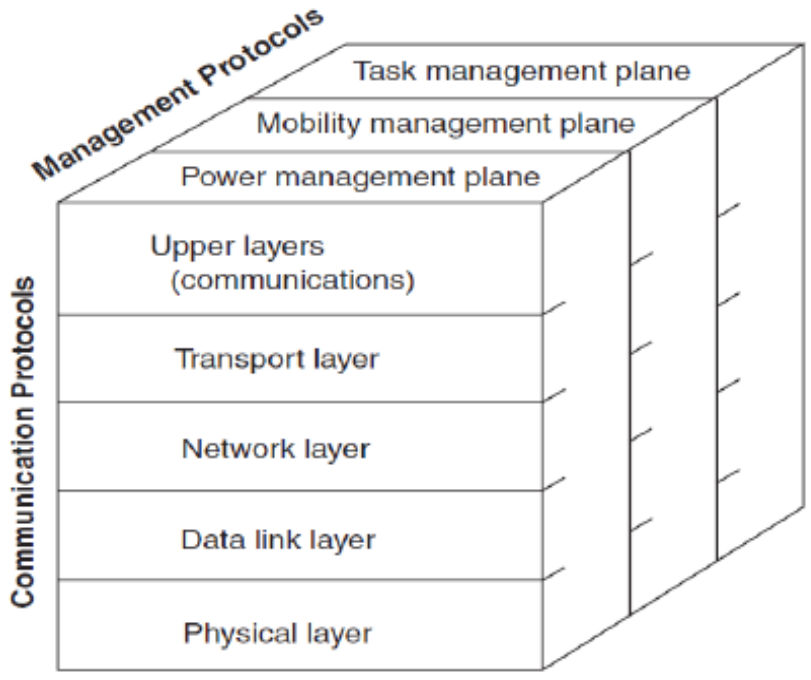

Figure.1 Protocol stack for WSN

\section{Background}

This section discusses about the existing research contribution of cross-layer based approach towards enhancing their communication performance of WSN. The studies carried out by Dien et al. [6] have emphasized working of Application layer, Network layer, Data link and Physical layer for Multimedia packet and path scheduling in WSN. Adoption of MAC layer and network layer for identification of network configuration to offer higher reliability has been carried out by Dobslaw et al. [7]. Nearly similar form of the work has been carried out by Park et al. [8]. Adoption of cross layer optimization using genetic algorithm towards solving scheduling, allocation of power, and data communication has been seen in the work of Yetgin et al. [9]. Modelling of cross layer for incorporating interaction among Physical, MAC, Network, and Application layer for enhancing the energy efficiency was developed by Jemeli et al. [10].

Lin et al. [11] have presented a cross-layer technique for enhancing the quality of service as well as energy efficiency in WSN. Pinto et al. [12] have used cross-layer approach for solving the delay problems for enhancing the data delivery services in WSN. Xu et al. [13] have presented a system model designed for cross layer using stochastic methodology for addressing issues related to energy management in WSN. Boubiche et al. [14] have used Cross layer approach for securing data aggregation using watermarking technique on heterogeneous WSN. Yetgin et al. [15] have presented cross layer approach for enhancing the network lifetime by emphasizing on the physical layers. 
Similar cross layer methodology was also used in the study of Lin and Kokkinos [16] for solving the hidden terminal problem. Cross layer scheme was also used for addressing the problem of retransmission as seen in the work carried out by Tseng and Chuang [17] using mathematical analysis. Wang et al. [18] have presented a queuing model using stochastic approach for implementing cross layer for minimizing delay. Shah et al. [19] have enhanced the quality of service by using MAC layer, link layer, network layer, and application layer. Issues associated with hidden terminal was addressed in the work carried out by Tseng et al. [20] where the author have presented a technique to detect as well as assign with no possibility of control overhead in WSN. Vuran and Akyildiz [21] have introduced a scheme focussing on MAC as well as other layers for ensuring effective data delivery practices in WSN.

Wang et al. [22] have presented an energy effective scheme using sleep scheduling approach especially focusing on service-oriented design in WSN. Melodia and Akyildiz [23] have presented similar approach toward ultra wide band sensor network using temporal hopping approach in heterogeneous WSN. Therefore, it can be seen that there has been significant number of research contribution focusing on using cross layer approach where majority of the layers have been used for addressing the existing problems as well as enhancing the communication system in WSN. However, it is also associated with set of issues that were found unsolved. The next section outlines such problems that have been identified in the proposed research work in order to address it.

\section{Research problem}

The problems identified to be addressed in the proposed research are as follows:

- Existing Cross-Layer based approaches doesn't balance the demands of real-time application with dynamic characteristic.

- Possibility of delay exists when multiple layers are involved with complex algorithm implementation that results in more end-toend delay.

- There is no ranking mechanism within the beacons to flag the importance of the either incoming or outgoing message in dynamic networks of WSN.

- The present technique of cross layer for energy efficiency considers very less inclusion of investigative parameters that result in effective energy modelling.
Therefore, the problem statement of the proposed study can be formulated as the design and development of a Cross-Layer Interactive Framework model that considers multiple potential dynamic attributes like end-to-end delay, message arrival duration and residual energy for enhancing energy efficiency and data delivery performance in WSN.

\section{Proposed solution}

The proposed system is an extension to our prior research implementation where we have emphasized on Spatio-Temporal scheme in Cross Layer design in WSN [24]. This part of the study implements an analytical model where a novel cross-layer scheme is designed for ensuring the compliance of real-time communication demands of WSN along with energy efficiency. The proposed Cross-Layer Interactive Framework (CLIF) Model is as shown below in Fig. 2.

The proposed CLIF model basically draws a communication bridge between Network and Data Link layer, where Data Link layer also consist of logical link layer and MAC layer. The proposed system extracts information of sleep and active duration from MAC layer in order to compute operational time with respect to complete time cycle. There are 4 different forms of time slots information that is used for performing provisioning of the messages that relates to broadcasting operation and linking operation. In order to cater up the real-time demands, a sensor node should offer preciseness in provisioning process by understanding the priority of the messages. For this purpose, a ranking mechanism is designed that offers high $(\mathrm{H})$, medium (M), and low (L) ranks on the messages and correspondingly it also adjust the buffer in similar fashion.

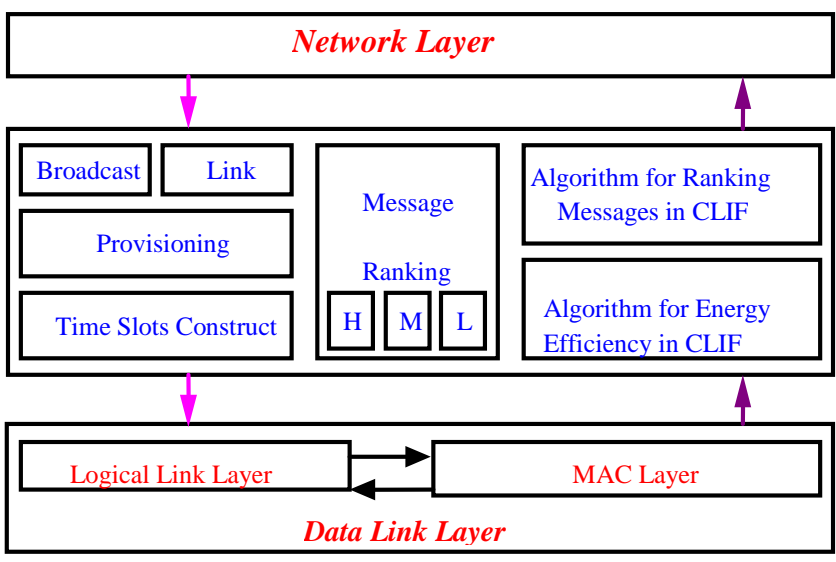

Figure.2 CLIF model 
After the neighbour nodes are discovered, the proposed system performs clustering using on the basis of the syncing status. A single hop list of adjacent nodes is constructed that is followed by allocation of time slots. The proposed system uses beacons of 32 bits that performs route request (RREQ) and route response (RREP). The significant contribution of CLIF is that i) it offers a buffering mechanism that is dynamically changed as per the need of ranks in the message leading to less effect of dynamic traffic load, ii) a significant enhancement energy modelling that is capable of computing energy depletion very precisely as it considers multiple parameters in energy depletion, iii) compensation of energy depletion due to cross layer interaction, and iv) lowered computational complexity owing to consideration of minimizing overheads due to multiple copies of beacons. Overall, CLIF offer energy efficiency with a good balance with data delivery performance. The next section outlines the algorithm implemented for this purpose.

\section{Algorithm for CLIF model}

The core agenda of the proposed algorithm implementation is to explicitly control the interaction between the Network layer and Link layer for the purpose of retaining better communication performance in terms of energy efficiency. The details of the algorithm involved in presented study are as follows:

While performing Cross-Layer Interaction, it is essential that each layer involved in the design process should yield faster response as a matter of processing the incoming task request. The algorithm takes the input of operational time and computes active period in data and MAC layer by multiplying it with frame-size. The duration of sleep is computed by (1-operational time) * frame size. The algorithm computes 4 different time slots $\left(T_{1} \rightarrow\right.$ syncing time, $T_{2} \rightarrow$ beacon exchange time, $T_{3} \rightarrow$ time of sleep after the transmission, $T_{4} \rightarrow$ time of sleep after the acknowledgement) on the basis of these active duration and sleep duration of a node and pass this information to the network layer.

The algorithm also takes the input of message arrival duration, and percentage of ranking for message. After obtaining all the hop information (Line-2), the algorithm applies first-order radioenergy model $\left(\phi_{T}, \phi_{R}\right)$ in order to compute transmittance energy $E_{t x}$ and receiving energy $E_{r x}$ (Line-3) considering Euclidean distance $d$ and packet length $p$. Once the MAC layer receives the provisioning information of broadcast and link, it attempts to obtain the information regarding total arrival of message $M_{\text {arr }}$, flag for transmitting dedicated slots $T_{\text {flag, }}$, and flag for receiving dedicated slots $R_{\text {flag }}$ (Line-4). Basically, we define dedicated slot as exclusive time slots between two communicating nodes one and a channel of receiving and transmission is constructed by the node by MAC layer being interacting with network layer. The significant steps of the algorithms are as follow:

The proposed algorithm uses a concept of ranking that offers incorporation of messages that are required to be ordered according the demands of applications.

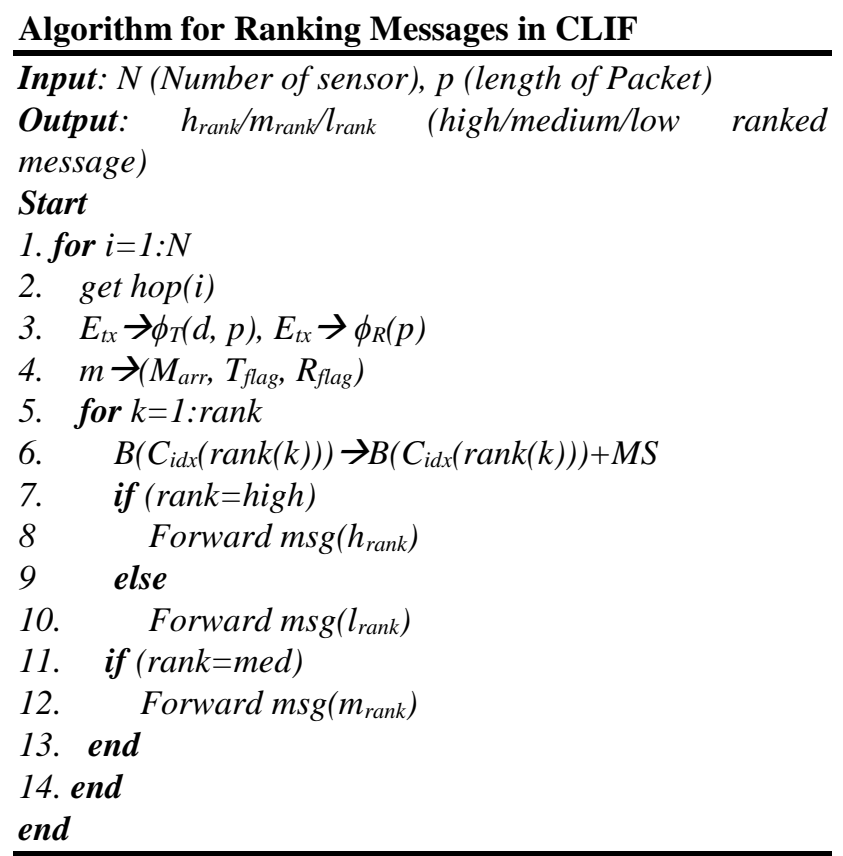

This information can be retrieved from application layer and can be redirected to network layer for undertaking decision of rank to be allocated. For all the possible number of allocated ranks (Line-5), the buffer $B$ is computed for all the cluster node $C_{i d x}$ and the buffer is controlled equivalently based on the ranks allocated for each source node $C_{i d x}$ belonging to any particular clusters. This information is accessed by the data link layer and is forwarded to the network layer which undertakes the decision of ranking the nodes based on higher ranking $h_{\text {rank, }}$, lower ranking $l_{\text {rank }}$, and medium ranking $m_{\text {rank }}$ (Line-7 to Line-12). Only the message with $h_{\text {rank }}$ is processed first followed by $m_{\text {rank }}$, and $l_{\text {rank }}$.

Apart from this, the data link layer and the MAC layer consistently exchanges message for updating the types of ranks involved in the message purpose so that energy could be controlled in specific manner. Another interesting fact of this algorithm is that it usages static memory (buffer) where older buffer information is replaced by new one. The size of such buffer can be directly dependent on the data obtained from application layer. Hence, the 
proposed algorithm offers a significant level of interaction among different layers of protocol stack of sensor node that suits well with the dynamic application of WSN using the cross layer interaction-based approach. The next algorithm applies modelling of energy computation.

A closer look into previous algorithm shows that it has used standard of first-order radio-energy modelling approach $\left(\phi_{T}, \phi_{R}\right)$ for computing transmittance and receiving energy. However, the applicability of this is limited only to node that doesn't much consider the level of interaction being carried out by different layers in protocol stack. Therefore, we introduce an algorithm that performs computation of actual energy being consumed by proposed cross-layer interaction. The steps involved in this algorithm are as follows:

\section{Algorithm for Energy Efficiency in CLIF}

Input: $N$ (Number of sensor)
Output: $E_{\text {tot }}$ (total energy)
Start
1. for $i=1: N$
2. $\quad E_{T X}\left(m_{1}, m_{2}\right) \rightarrow \sum_{i=1}^{4} E_{i .}\left(T_{1}+T_{2}+T_{3}+T_{4}\right)$
3. $E_{R X}\left(m_{1}\right) \rightarrow \sum_{i=1}^{4} E_{i .}\left(T_{1}+T_{2}+T_{3}+T_{4}\right)$
4. $E_{R X}\left(m_{2}\right) \rightarrow \sum_{i=1}^{4} E_{i .}\left(T_{1}+T_{3}+T_{4}\right)$
5. $E_{t o t} \rightarrow \sum_{i=1}^{8} \operatorname{var}$
end

Before initiating this algorithm, the system initially groups all the messages with respect to identifies of transmittance and receiving of dedicated time slots $\left(T_{\text {flag }}\right.$ and $R_{\text {flag }}$ ) and performs summation of it with respect to the link and broadcasting scheduling. This computation of energy is carried out for all the nodes (Line-1). The algorithm initially computes the transmittance energy $E_{T X}$ considering mode of broadcast $\left(m_{1}\right)$ and link provisioning $\left(m_{2}\right)$ (Line-2). A closer look into expression in Line-2 shows that there are four components associated with time slots i) maximum $E_{T X}$ value multipled with $T_{1}$, ii) $E_{R X}$ value multipled with $T_{2}$, iii) average $E_{T X}$ value with medium ranks multipled with $T_{3}$, and iv) $E_{R X}$ value multipled with $T_{4}$. The algorithm also performs the similar computation for two different scheduling modes $m_{1}$ and $m_{2}$ for $E_{R X}$ as shown in Line-3 and Line-4. The algorithm also computes the total value of the energy being consumed $E_{t o t}$ as shown in Line5.

The data structure used to apply this algorithm is priority queue. There are 8 different variables var involved in this step i.e. i) $\operatorname{ETX}(m 1) * \operatorname{ETX}(m 2)$, ii) sum of all transmittance schedules of broadcast multiplied with energy required at transmitting link schedules, iii) sum of all receiving schedules of broadcast multiplied with energy required at receiving link schedules, iv) sum of all receiving schedules of link multiplied with energy required at receiving at broadcast schedules, v) overheads, vi) idle time and energy at channel, vii) sleep time and energy at channel, and viii) initialized transition time of transmission and energy. The algorithm performs the computation of overhead that could possibly cause due to beacon exchanging between the nodes as well as internal exchange of control message among the layers. For this purpose, overhead is computed by subtracting all the sum of message arrival time greater than zero with number of receiving broadcast scheduling. This process is carried out exclusively for route request beacons.

The algorithm also computes the overhead that could possibly be raised from route response beacons by subtracting all the sum of message arrival time greater than zero with number of receiving link schedules. Finally, both the overhead computed for route request and response is added up in order to obtain cumulative overhead arising. An interesting fact to observe in the proposed algorithm is that it offers a streamlined mechanism of computing the cumulative energy considering all the possible cases of link as well as broadcast schedules that are controlled mainly in the link layer. As the link layer directly communicates with the network layer, therefore all the updated information about the network statistics is computed and shared between link and network. The complete energy modelling is carried out considering possible overheads along with idle listening.

The algorithm assumes that energy is not much consumed when the node is in sleep state whereas there is a specific range of energy consumed when the state of the sleep is changed to active state. As the proposed technique considers both link and broadcast provisioning technique, it performs precise computation of energy consumption considering the case study of presence of and absence of dedicated time slots. This evaluation was carried out for both transmittance and receiving energy. Hence, the proposed technique of CLIF offers a fine tuning of transmittance energy, where energy is not only computed by also minimized. The proposed technique also offers a comprehensive communication mechanism among the data link and MAC layer within link layer that also performs communication with the network layer in dynamic traffic condition of WSN. The study outcome of the proposed CLIF is briefed in the next section. 


\section{Result analysis}

The complete design of CLIF is carried out on Matlab considering random distribution of the sensor nodes of 100-1000 dispersed over 1000x1100 $\mathrm{m}^{2}$ simulation area. The Simulation parameters considered for the proposed research work is presented in Table 1. The study outcome is compared with similar form of work recently carried out by Khanh et al. [25] and Liang [26]. The work carried out by Khanh et al. introduced a model called as RP-MAC that is meant for overcoming the problems associated with idle listening time while using conventional layered approach. While, the work carried out by Liang presented a model called as ERCO that have used cross layer approach by integrating routing layer and MAC layer in order to accomplish energy efficiency.

In the proposed research work to develop a robust CLIF model, operational time is taken into consideration. Operational Time (OT) is defined as the time period during which the network is operational. In order to simulate CLIF and for effective Cross-Layer interaction computing four time slots namely $\mathrm{T} 1, \mathrm{~T} 2, \mathrm{~T} 3$ and $\mathrm{T} 4$ becomes significant. For the differing values of OT, their corresponding time slots are tabulated in Table 2 below.

The relevance of the developed CLIF model is in accordance with the QoS parameters like Operational time, end-to-end delay, energy consumed per bit, residual energy and inter arrival time of the message.

Table 1. Simulation parameters

\begin{tabular}{|l|l|}
\hline \multicolumn{2}{|c|}{ Simulation Environment Parameters } \\
\hline Simulator & Matlab R2015a \\
\hline Network Type & Wireless \\
\hline No. of nodes & $100-1000$ \\
\hline Area & $1000 x 1100 \mathrm{~m}^{2}$ \\
\hline Communication radius & $\begin{array}{l}\text { Determined by } 8 \\
\text { neighbouring nodes }\end{array}$ \\
\hline Sink node location & $\begin{array}{l}\text { Varying in the area } \\
1000 x 1100 \mathrm{~m}^{2}\end{array}$ \\
\hline Mobility pattern & Circular \\
\hline No. of clusters & $1-10$ \\
\hline Operational Time & $0.05-0.5$ \\
\hline Message arrival duration & $1-10 \mathrm{sec}$ \\
\hline Initial Energy & $50 \mathrm{~mJ}$ \\
\hline Transmission Energy & $0.1 \mathrm{~mJ}$ \\
\hline Receiving Energy & $0.1 \mathrm{~mJ}$ \\
\hline Message rank & High (H) Medium (M), \\
Low $(\mathrm{L})$
\end{tabular}

Table 2. Operational time parameter

\begin{tabular}{|l|l|}
\hline Operational Time & Time Slot \\
\hline \multirow{5}{*}{$50 \mu \mathrm{s}$ (Minimum) } & $\mathrm{T} 1-10 \mu \mathrm{s}$ (Sync) \\
\cline { 2 - 2 } $275 \mu \mathrm{s}$ (Average) & $\mathrm{T} 2-40 \mu \mathrm{s}$ (Beacon) \\
\cline { 2 - 2 } & $\mathrm{T} 3-760 \mu \mathrm{s}$ (Trans-sleep) \\
\cline { 2 - 2 } $500 \mu \mathrm{s}$ (Max) & $\mathrm{T} 4-190 \mu \mathrm{s}$ (ACK-sleep) \\
\hline & $\mathrm{T} 1-10 \mu \mathrm{s}$ (Sync) \\
\cline { 2 - 2 } & $\mathrm{T} 2-40 \mu \mathrm{s}$ (Beacon) \\
\cline { 2 - 2 } & $\mathrm{T} 3-760 \mu \mathrm{s}$ (Trans-sleep) \\
\cline { 2 - 2 } & $\mathrm{T} 4-190 \mu \mathrm{s}$ (ACK-sleep) \\
\hline & $\mathrm{T} 1-55 \mu \mathrm{s}$ (Sync) \\
\cline { 2 - 2 } & $\mathrm{T} 2-22 \mu \mathrm{s}$ (Beacon) \\
\cline { 2 - 2 } & $\mathrm{T} 3-58 \mu \mathrm{s}$ (Trans-sleep) \\
\cline { 2 - 2 } & $\mathrm{T} 4-145 \mu \mathrm{s}$ (ACK-sleep) \\
\hline
\end{tabular}

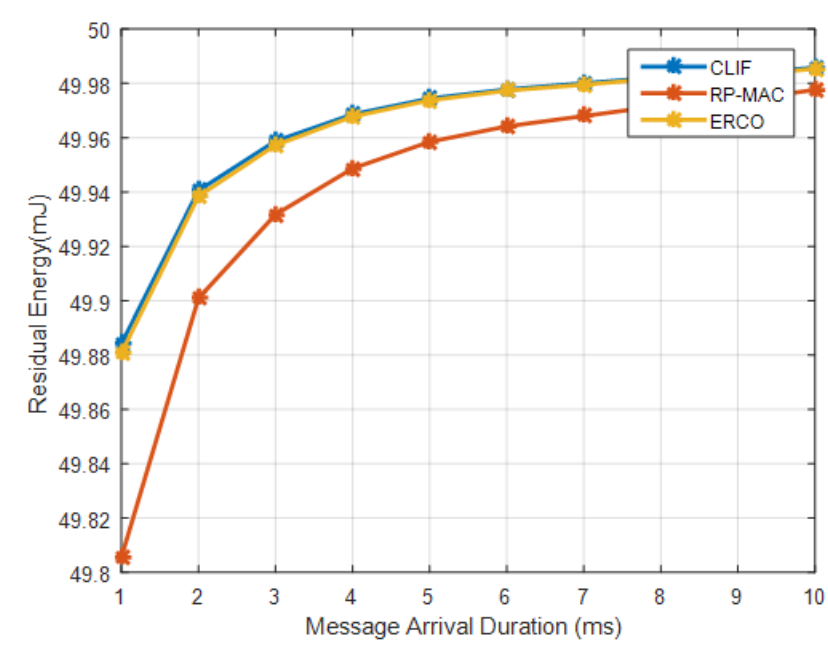

Figure. 3 Inter arrival time with the residual energy

Inter arrival time is described as the time between two successfully arriving data packets at the MAC layer. The cuurent value of energy in a node after the reception or transmission of routing packets is the residual energy. The transmission energy $\mathrm{E}_{\mathrm{TX}}$ is calculated by considering link provisioning and mode of broadcast based on the message being ranked. The graph in Fig. 3 illustrates that the energy efficiency of the proposed CLIF is $99.92 \%$ which excels better when compared with the energy efficiency of the existing approaches of ERCO - 97.76\% and RP-MAC - 95.6\%. The contribution of the CLIF shows increase of $2.16 \%$ with respect to well-known ERCO cross-layer approach and $4.32 \%$ increase with the layered approach.

End-to-end delay or one-way delay (OWD) refers to the time consumed by a packet to be transmitted across a network from intended source to destination. In Fig. 4, end-to-end delay in CLIF is $83.3 \%$, there is a considerable decrease in the delay when compared with ERCO having delay $86.6 \%$ and RP-MAC with $98.3 \%$. The delay in the implemented work is reduced eventually and CLIF can be considered for applications which require less delay time. The reduction with respect to delay 


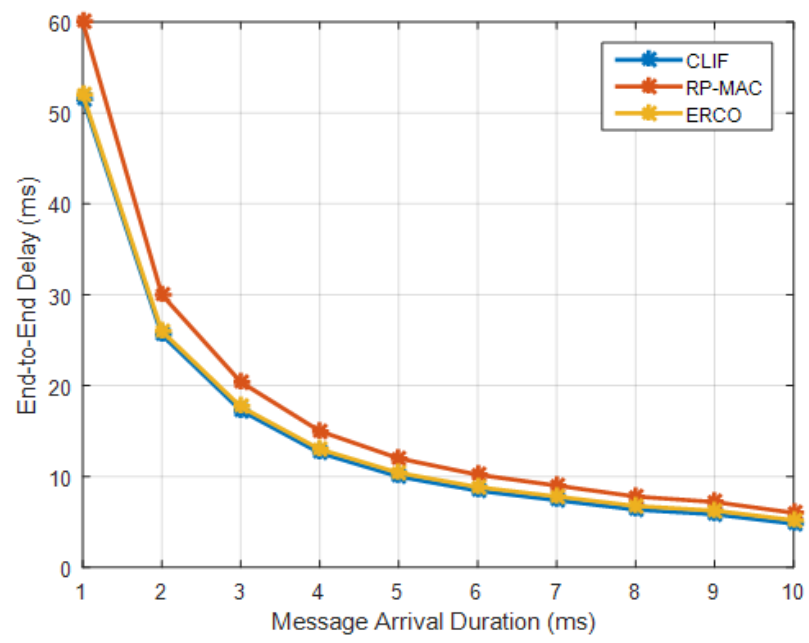

Figure.4 Inter arrival time with end-to-end delay

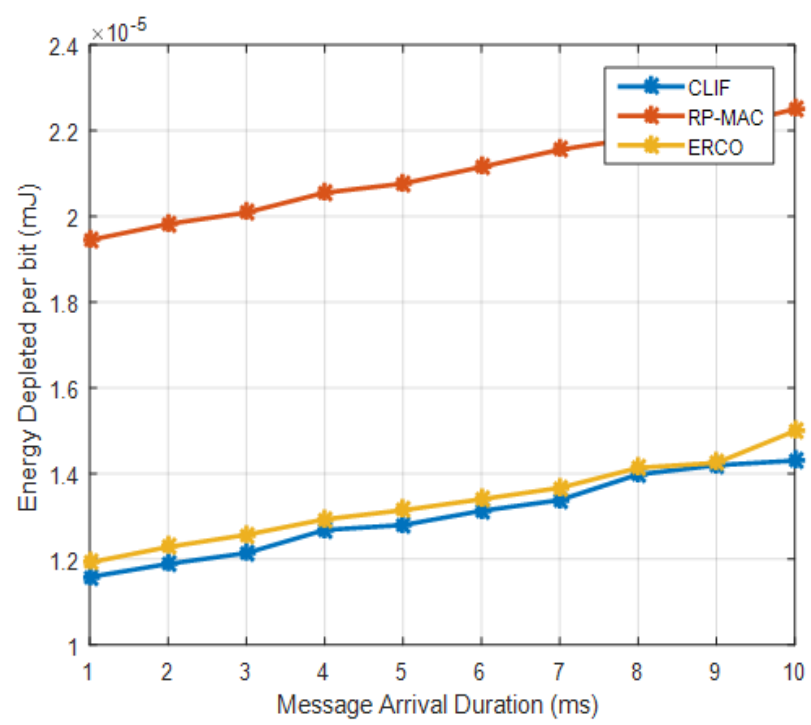

Figure.5 Inter arrival time with energy depleted per bit

is achieved by scheduling the link and incorporating necessary provisions in broadcasting the message due to the implications of the time slots used in the duty cycle. The delay in the data delivery is reduced substantially by $3.3 \%$ with respect to ERCO crosslayer approach and by $15 \%$ when compared to the traditional layered approach.

In Fig. 5, the energy depletion in taken into account with the message being transmitted in bits which arrives in the range 1-10ms. Energy consumption for transmitting the message per bit is reduced due to the interaction between the MAC layer and the Network layer. The information which is passed on to the higher layer from lower layer includes when the broadcasting is to be scheduled for the messages being prioritized and also link provisioning based on the time slots supplied to each of the deployed node in the network, which considerably helps in the diminished energy consumption which increases the overall operational time of the netwok. The

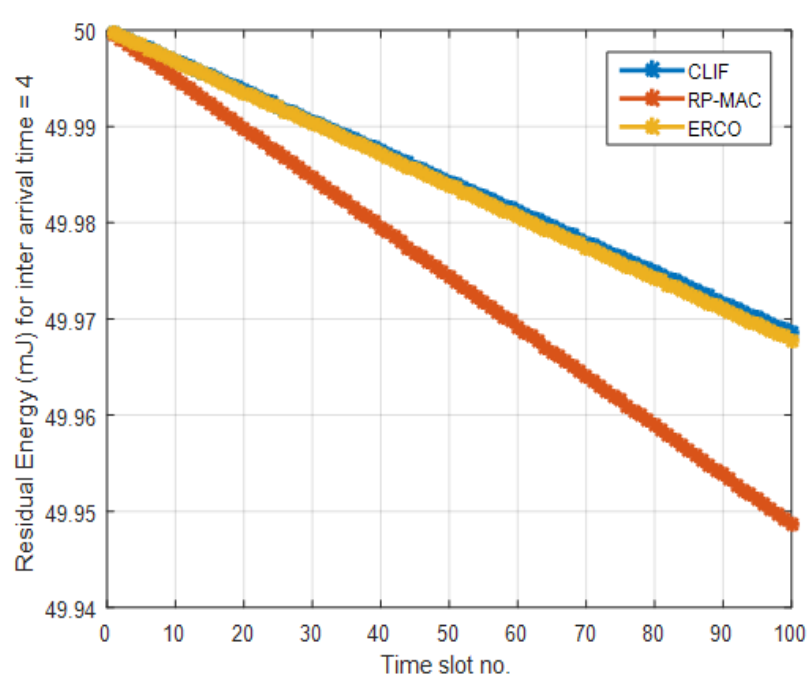

Figure.6 Time slot with residual energy

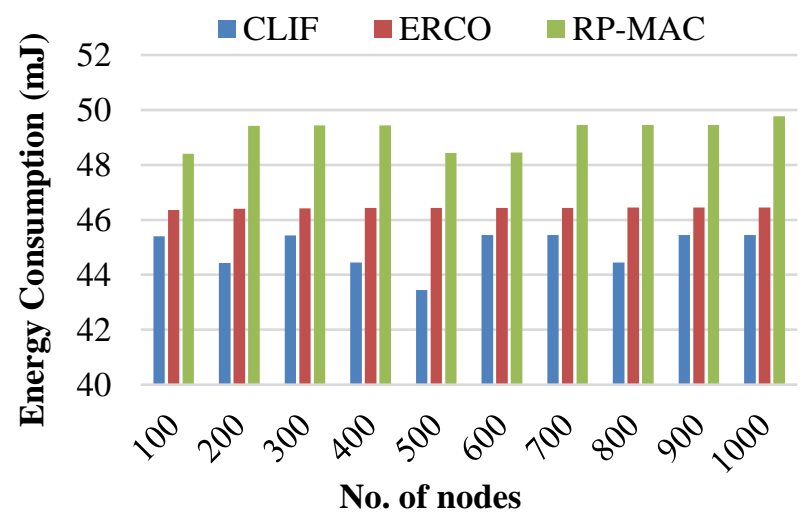

Figure.7 No. of nodes with energy consumption

graph depicts that the proposed work results to $47.75 \%$ in terms of energy depletion. Comparatively the ERCO results in $50 \%$ of energy depletion and RP-MAC results in $83.3 \%$ energy utilization per bit. The inference is that CLIF reduces energy exploitation by $2.25 \%$ when compared to ERCO and by $34.5 \%$ with RP-MAC.

For the graph in Fig. 6, the residual energy is compared with the time slots. The time slot considered is in the range 0 to 100 . Introducing the concept of time slot inorder to schedule the link provisioning enhances the energy consumption of the overall network and also increases the efficiency in terms of network lifetime. The time slots are computed to take decidability account with respect to how many nodes need to be active during transmission and how many nodes can exist in sleep mode after transmission of ACK and the message.

The simulation is carried out for nodes ranging from 100 to 1000 with initial energy for all the nodes as $50 \mathrm{~mJ}$. The graph of Fig. 7 shows that the highest energy consumption in CLIF is $45.3 \mathrm{~mJ}$ whereas in ERCO cross-layer approach it is $46.2 \mathrm{~mJ}$ 
and in RP-MAC it shows a maximum energy consumption of 49.2MJ. The observations from the graph also reveal that ERCO approach has a stable energy consumption in all rounds, whereas in CLIF the energy consumption is varied as the messages are ranked and only high priority messages need to be transmitted first. Hence the proposed CLIF model outperfors the existing cross-layer and conventional layered approach and the entire framework can be used for deploying diverse IoT applications.

The most prominent factor of CLIF is that we can determine the active nodes and sleep nodes for the various operational time and the deployed nodes. The CLIF model implemented considers only less no. of nodes to be active to do the data transmission by considering the dynamic characteristics of the traffic generated by the sensor node. Further investigation on the graph in Fig. 8 reveals that when the number of deployed nodes are 1000 and more, an equal probability of $50 \%$ nodes will be both in active mode and sleep mode. This inference will enhance the energy efficiency and faster communication within the network which improves the overall network lifetime. Additionally another advantage of CLIF is that the model can be used as a framework to set the deployed nodes in active and sleep mode which will be very much beneficial for time-critical, real-time, healthcare, smart-city, etc. applications.

The outcome clearly indicates that the proposed CLIF offers reduced energy consumption and reduced delay as compared to existing cross-layer based approach and layered approach. Although, the outcome of CLIF and ERCO bears closest resembles, still CLIF is considered as better version as it takes a highly comprehensive strategy into consideration to select energy efficient path with lower overhead which is missing in ERCO. Hence ERCO offers high computational complexity, whereas proposed system CLIF offers faster response time and enhanced energy utilization.

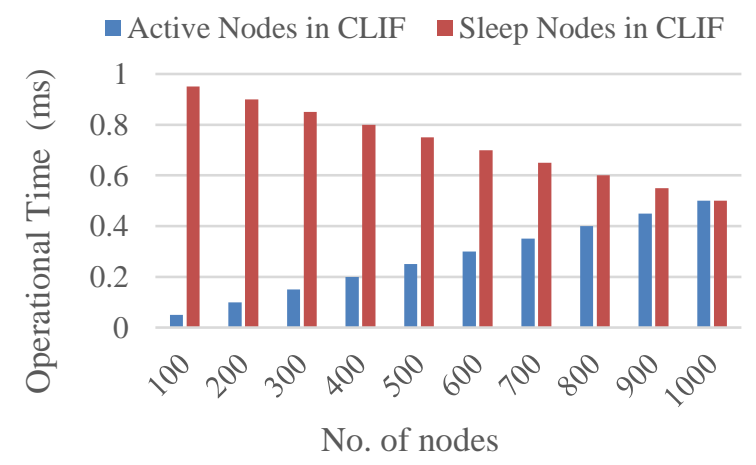

Figure. 8 No. of nodes with operational time

\section{Conclusion and future work}

The proposed research work have introduced the concept of CLIF in which Network layer and Data link layer has been taken into consideration to offer interaction among each other. CLIF incorporates a mechanism where all the incoming dynamic messages can be filtered as per their level of importance. This concept allows the applicability of CLIF to work on various time and mission critical applications in WSN in real-time environment. At the same time, there is no significant computational complexity associated with CLIF execution as it enhances the radio-energy modelling to incorporate more number of parameters to offer better computation and consumption of the energy, an important resource. Hence, the proposed system in true sense offers interaction among the layers offering to compensate the delay that could have possibly risen owing to bottleneck condition of traffic in WSN. Our future work will be in direction of further enhancing and optimizing the CLIF model to offer better communication services in WSN and IoT applications.

\section{Acknowledgments}

The authors would like to thank REVA University, School of Computing \& IT and Sri Venkateshwara College of Engineering, Bangalore, India for the constant support during the research work. The authors would also extend appreciation to their family members and friends for their generous support all through this research work.

\section{References}

[1] M. Asif, S. Khan, R. Ahmad, M. Sohail, and D. Singh, "Quality of Service of Routing Protocols in Wireless Sensor Networks: A Review", IEEE Journals \& Magazines, Vol. 5, pp. 18461871, 2017.

[2] I. Harish and S. I. Sambasivan, "A protocol stack design and implementation of wireless sensor network for emerging application", In: Proc. of the IEEE International Conference ON Emerging Trends in Computing, Communication and Nanotechnology, Tirunelveli, pp. 523-527, 2013.

[3] C. H. Feng, I. Demirkol, and W. B. Heinzelman, "UPS: Unified Protocol Stack for wireless sensor networks", In: Proc. of the 6th Annual International Mobile and Ubiquitous Systems: Networking \& Services, MobiQuitous, Toronto, ON, pp. 1-2, 2009. 
[4] Q. Wang, and I. Balasingham, "Wireless Sensor Networks - An Introduction", Y. K. Tan, Wireless Sensor Networks: Application-Centric Design, InTech, pp.1-13, 2010

[5] Manpreet and J. Malhotra, "A survey on cross layer routing protocols for manet", In: Proc. of the 2014 International Conference on Advances in Engineering \& Technology Research, Unnao, pp. 1-3, 2014.

[6] M. E. E. D. Abd El Kader, A. A. A. Youssif, and A. Z. Ghalwash, "Energy Aware and Adaptive Cross-Layer Scheme for Video Transmission Over Wireless Sensor Networks", IEEE Sensors Journal, Vol. 16, No. 21, pp. 7792-7802, 2016.

[7] F. Dobslaw, T. Zhang, and M. Gidlund, "QoSAware Cross-Layer Configuration for Industrial Wireless Sensor Networks" IEEE Transactions on Industrial Informatics, Vol. 12, No. 5, pp. 1679-1691, 2016.

[8] P. Park, P. D. Marco, and K. H. Johansson, "Cross-Layer Optimization for Industrial Control Applications Using Wireless Sensor and Actuator Mesh Networks", IEEE Transactions on Industrial Electronics, Vol. 64, No. 4, pp. 3250-3259, 2017.

[9] H. Yetgin, K. T. K. Cheung, M. El-Hajjar, and L. Hanzo, "Network-Lifetime Maximization of Wireless Sensor Networks", IEEE Access, Vol. 3, pp. 2191-2226, 2015.

[10] M. Al-Jemeli and F. A. Hussin, "An Energy Efficient Cross-Layer Network Operation Model for IEEE 802.15.4-Based Mobile Wireless Sensor Networks", IEEE Sensors Journal, Vol. 15, No. 2, pp. 684-692, 2015.

[11] S. C. Lin, I. F. Akyildiz, P. Wang, and Z. Sun, "Distributed Cross-Layer Protocol Design for Magnetic Induction Communication in Wireless Underground Sensor Networks", IEEE Transactions on Wireless Communications, Vol. 14, No. 7, pp. 40064019, 2015.

[12] P. Pinto, A. Pinto, and M. Ricardo, "CrossLayer Admission Control to Enhance the Support of Real-Time Applications in WSN", IEEE Sensors Journal, Vol. 15, No. 12, pp. 6945-6953, 2015.

[13] W. Xu, Y. Zhang, Q. Shi, and X. Wang, "Energy Management and Cross Layer Optimization for Wireless Sensor Network Powered by Heterogeneous Energy Sources", IEEE Transactions on Wireless Communications, Vol. 14, No. 5, pp. 28142826, 2015.
[14] D. E. Boubiche, S. Boubiche, and A. Bilami, "A Cross-Layer Watermarking-Based Mechanism for Data Aggregation Integrity in Heterogeneous WSNs", IEEE Communications Letters, Vol. 19, No. 5, pp. 823-826, 2015.

[15] H. Yetgin, K. T. K. Cheung, M. El-Hajjar, and L. Hanzo, "Cross-layer network lifetime optimisation considering transmit and signal processing power in wireless sensor networks", IET Wireless Sensor Systems, Vol. 4, No. 4, pp. 176-182, 2014

[16] C. K. Lin and T. Kokkinos, "Cross-Layer Solutions for Extended-Range Wireless Sensor Networks", IEEE Sensors Journal, Vol. 13, No. 3, pp. 1044-1054, 2013.

[17] H. W. Tseng and Y. R. Chuang, "A CrossLayer Judgment Scheme for Solving Retransmission Problem In: IEEE 802.15.4Based Wireless Body Sensor Networks", IEEE Sensors Journal, Vol. 13, No. 8, pp. 3124-3135, 2013.

[18] Y. Wang, M. C. Vuran, and S. Goddard, "Cross-Layer Analysis of the End-to-End Delay Distribution in Wireless Sensor Networks", IEEE/ACM Transactions on Networking, Vol. 20, No. 1, pp. 305-318, 2012.

[19] G. A. Shah, W. Liang, and O. B. Akan, "CrossLayer Framework for QoS Support in Wireless Multimedia Sensor Networks", IEEE Transactions on Multimedia, Vol. 14, No. 5, pp. 1442-1455, 2012.

[20] H. W. Tseng, S. C. Yang, P. C. Yeh, and A. C. Pang, "A Cross-Layer Scheme for Solving Hidden Device Problem in IEEE 802.15.4 Wireless Sensor Networks", IEEE Sensors Journal, Vol. 11, No. 2, pp. 493-504, 2011.

[21] M. C. Vuran and I. F. Akyildiz, "XLP: A Cross-Layer Protocol for Efficient Communication in Wireless Sensor Networks", IEEE Transactions on Mobile Computing, Vol. 9, No. 11, pp. 1578-1591, 2010.

[22] J. Wang, D. Li, G. Xing, and H. Du, "CrossLayer Sleep Scheduling Design in ServiceOriented Wireless Sensor Networks", IEEE Transactions on Mobile Computing, Vol. 9, No. 11, pp. 1622-1633, 2010.

[23] T. Melodia and I. F. Akyildiz, "Cross-layer QoS-aware communication for ultra wide band wireless multimedia sensor networks", IEEE Journal on Selected Areas in Communications, Vol. 28, No. 5, pp. 653-663, 2010.

[24] S. Chandra, S. Talanki, and K.K. Patil, "Integrated S-AODV and DEL-CMAC Algorithm of Spatio Temporal Cross-Layer in Sensor Network", Silhavy R., Senkerik R., 
Kominkova Oplatkova Z., Prokopova Z., Silhavy P. (eds) Cybernetics and Mathematics Applications in Intelligent Systems. CSOC 2017. Advances in Intelligent Systems and Computing, Vol. 574, Springer, 2017.

[25] Y. Liang, "Energy-efficient, reliable cross-layer optimization routing protocol for wireless sensor network", In: Proc. of the International Conference on Intelligent Control and Information Processing, Dalian, pp. 493-496, 2010.

[26] H. S. Khanh, C. Y. Ock, and M. K. Kim, "RPMAC: A cross-layer duty cycle MAC protocol with a Reduced Pipelined-forwarding feature for Wireless Sensor Networks", In: Proc. of the International Wireless Communications and Mobile Computing Conference, Dubrovnik, pp. 1469-1474, 2015. 